\title{
PROJEÇÃO ROBOTIZADA DE MASSA REFRATÁRIA PARA PROTEÇÃO DOS STAVES DO ALTO-FORNO 2 DA THYSSENKRUPP CSA *
}

\author{
Eustáquio Vieira Júnior ${ }^{1}$ \\ Sandro Valério Maia Larrubia ${ }^{2}$ \\ Dalton Martins $\mathrm{Neto}^{3}$ \\ Alexandre Patrício Vieira da Silveira ${ }^{4}$ \\ Bruno Pinheiro da Silva ${ }^{5}$
}

\section{Resumo}

O Alto-Forno 2 da thyssenkrupp CSA, no Rio de Janeiro, iniciou sua primeira campanha em 16 de dezembro de 2010. No decorrer de sua campanha, verificou-se problemas de deslocamento de staves de ferro fundido, deformação dos staves de cobre e desgaste acentuado do refratário do top cone. $\mathrm{Na}$ análise dos eventos adotou-se a inspeção por vídeo endoscopia e em seguida foram tomadas algumas ações para recuperação e preservação do Alto-Forno 2, sendo estendidas também do Alto-Forno 1. Dentre as ações de preservação dos Altos-Fornos destaca-se a projeção robotizada de material refratário, shotcrete, visando reduzir o desgaste acentuado dos staves e deterioração de suas tubulações de refrigeração. $O$ trabalho apresenta o detalhamento das técnicas utilizadas e seleção de materiais assim como os preparativos operacionais que garantiram o bom desempenho do AltoForno 2 nos momentos que antecederam a parada e após seu retorno à operação, mostrando que a atividade é viável do ponto de vista técnico e financeiro.

Palavras-chave: Alto-Forno; Staves; Shotcrete; Refrigeração.

\section{REFRACTORY MASS PROJECTION TO PROTECT THE STAVE COOLERS OF BLAST FURNACE\#2 - THYSSENKRUPP CSA PLANT}

\section{Abstract}

The thyssenkrupp CSA Blast Furnace\#2, in Rio de Janeiro, begins to operate in 16 of December, 2010. In this campaign happened: the cast iron staves displacement, deformation in copper staves and strong wear in refractory layer of top cone. In the analysis, process, in 2016, uses the video endoscopy and in sequence, some actions are used to preserve and recover the Blast Furnace\#2, and in the future in Blast Furnace\#1. Among the actions to preserve the Blast Furnaces, the shotcrete action has a great attention because try to reduce the wear in staves and the damaged in the water pipes. The report shows the technics, materials selection, application procedure and others before and after the shocrete and the results show that this activity is viable of technical and economic point of view.

Keywords: Blast Furnace; Staves; Shotcrete; Coolling system.

1 Engenheiro Metalurgista, Gerência de Operação dos Altos-Fornos, thyssenkrupp CSA, Rio de Janeiro, RJ, Brasil.

2 Engenheiro de Produção, Gerência de Operação dos Altos-Fornos, thyssenkrupp CSA, Rio de Janeiro, RJ, Brasil

3 Engenheiro Mecânico, Coordenador de Preservação dos Altos-Fornos, Gerência de Operação dos Altos-Fornos, thyssenkrupp CSA, Rio de Janeiro, RJ, Brasil

4 Técnico Especialista de Processos, Gerência de Operação dos Altos-Fornos, thyssenkrupp CSA, Rio de Janeiro, RJ, Brasil

5 Engenheiro Químico, Gerência Técnica de Redução, thyssenkrupp CSA, Rio de Janeiro, RJ, Brasil. 


\section{INTRODUÇÃO}

A usina siderúrgica da thyssenkrupp CSA, no Rio de Janeiro-RJ, apresenta dois altos-fornos, estes Altos-Fornos têm mesma capacidade produtiva, o Alto-Forno $1 \mathrm{e}$ o Alto-Forno 2 entraram em operação em junho e dezembro 2010, respectivamente. Os Altos-Fornos da thyssenkrupp CSA apresentam sistema de refrigeração por stave-coolers no corpo e jaqueta no cadinho e os regeneradores são de câmara de combustão externa[1].

As ocorrências de início de operação dos Altos-Fornos e da planta da thyssenkrupp CSA, trouxeram diversas instabilidades operacionais agravadas ao grande número de paradas sequenciais das unidades produtivas. Visto que estas situações acarretaram consequências nos Altos-Fornos, foi estabelecida nova diretriz para ações de preservação destes equipamentos, objetivando restabelecer as condições operacionais e estender a vida útil dos Altos-Fornos e seus equipamentos periféricos.

Os staves dos Altos-Fornos da thyssenkrupp CSA, são de cobre entre a primeira fileira da rampa até a quarta fileira que está na cuba inferior e são de ferro fundido da quinta fileira que está na cuba inferior até a oitava fileira que está na cuba superior.

No presente trabalho são apresentadas todas etapas vivenciadas para execução do shotcrete na região da cuba do Alto-Forno 2, o planejamento para shotcrete, os aspectos da projeção propriamente dita e os resultados obtidos após a aplicação do refratário.

As principais características do Alto-Forno 2 da thyssenkrupp CSA são mostradas na tabela 1 e os principais resultados operacionais da sua campanha são mostrados na tabela 2.

Tabela 1. Principais características do Alto-Forno 2 da thyssenkrupp CSA

\begin{tabular}{|c|c|c|}
\hline Itens & Unidade & AF2 \\
\hline Volume interno & $\mathrm{m}^{3}$ & 3284 \\
\hline Volume de trabalho & $\mathrm{m}^{3}$ & 2775 \\
\hline Diâmetro do cadinho & $\mathrm{m}$ & 12,0 \\
\hline Capacidade de produção & t/dia & 7500 \\
\hline Número de ventaneiras & - & 32 \\
\hline Número de furo de gusa & - & 2 \\
\hline Topo & - & $\begin{array}{c}\text { Topo sem } \\
\text { cone }\end{array}$ \\
\hline Sistema de granulação & - & INBA \\
\hline Temperatura máxima de sopro & $\stackrel{\circ}{ } \mathrm{C}$ & 1250 \\
\hline $\begin{array}{c}\text { Sistema de refrigeração cuba, } \\
\text { ventre e rampa }\end{array}$ & - & Stave-coolers \\
\hline $\begin{array}{c}\text { Sistema de refrigeração do } \\
\text { cadinho }\end{array}$ & - & Jaqueta \\
\hline Início da campanha atual & - & $16 / 12 / 2010$ \\
\hline
\end{tabular}


Tabela 2. Dados da Campanha do Alto-Forno 2 da thyssenkrupp CSA

\begin{tabular}{|c|c|c|c|c|c|c|c|c|}
\hline \multirow{2}{*}{ CAMP* $^{*}$} & BLOW IN & BLOW OUT & \multirow{2}{*}{$\begin{array}{c}\text { PRODUTIVIDADE } \\
\mathrm{t} / \mathrm{d} / \mathrm{m}^{3} \\
\end{array}$} & CR & ÓLEO & \multirow{2}{*}{ PCI } & \multirow{2}{*}{\multicolumn{2}{|c|}{ FR }} \\
\hline & \multicolumn{2}{|c|}{ D A T A } & & \multirow{2}{*}{\multicolumn{5}{|c|}{$\mathrm{kg} / \mathrm{t}$}} \\
\hline \multicolumn{5}{|c|}{ ALTO-FORNO 2} & & & & \\
\hline $1^{\underline{a}}$ & $16 / 12 / 2010$ & - & 1,47 & 419 & - & 107 & - & 525 \\
\hline
\end{tabular}

*Número da campanha

\section{MATERIAIS E MÉTODOS}

\subsection{Desgaste dos Refratários, Deslocamento e Deformação dos Staves}

A partir de 2016, foram identificadas algumas regiões com desgaste. Estas regiões, ilustradas na figura 1, eram nos refratários da cúpula do topo do Alto-Forno 2 e nos staves cooler da cuba inferior. Os staves danificados estavam concentrados na região da última fileira de cobre e da primeira de ferro fundido

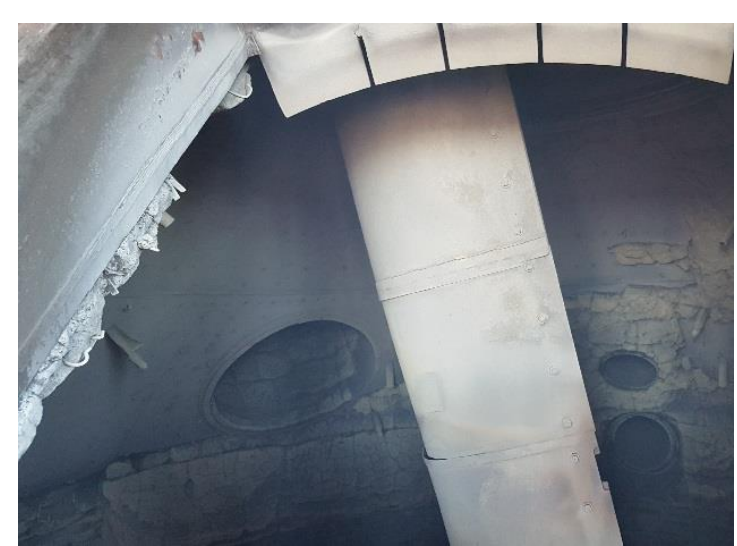

a) Cúpula do topo

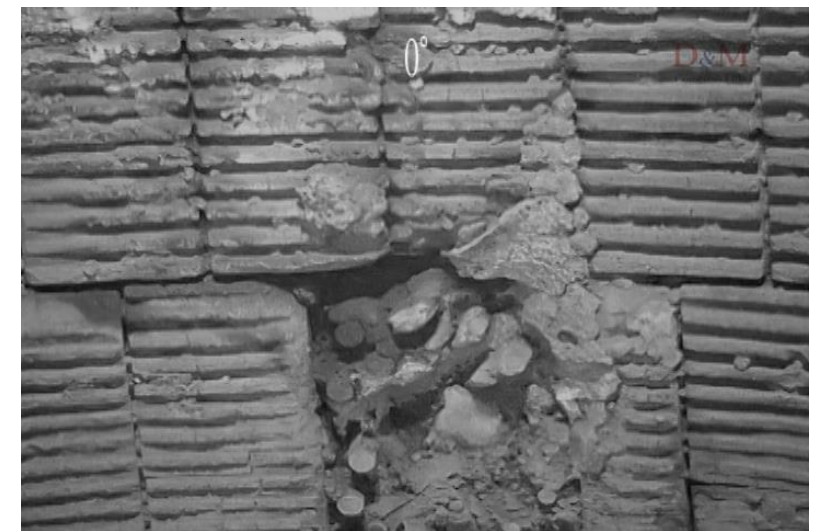

b) Cuba inferior com desgaste nos staves

Figura 1. Inspeção das regiões desgastadas em 2016

As origens dos problemas encontrados foram identificadas como:

- Excesso de temperatura do gás de topo, causando desgaste dos refratários;

- Movimentação dos staves de ferro fundido com rompimento dos parafusos de fixação;

- Deformação dos staves de cobre devido excesso de carga térmica durante a operação;

\subsection{Metodologia de Reparo e Preservação}

O plano de preservação dos staves estabelece as seguintes ações, nesta ordem sequencial:

- resfriamento da carcaça com filme fino de água; 
- instalação de cigar coolers para recuperar a refrigeração perdida por tubos danificados;

- inserção de mangotes flexíveis;

- injeção de massa refratária;

- realização de shotcrete;

- troca de staves mais danificados;

Dentre as ações descritas acima, o shotcrete era o próximo passo do planejamento a ser realizado. Portanto, em 2016, foram iniciados os preparativos para a execução da aplicação robotizada de refratário.

Os materiais selecionados de acordo com a região de aplicação são mostrados na tabela 3

Tabela 3. Materiais utilizados no shotcrete do Alto-Forno 2

\begin{tabular}{c|c}
\hline Região do forno & Material \\
\hline Cúpula do topo & Concreto de baixo cimento a base de mulita \\
\hline Coroa de choque e cuba superior & $\begin{array}{c}\text { Material de baixo cimento a base de alumina } \\
\text { e carbeto de silício }\end{array}$ \\
\hline Cuba superior e cuba inferior & $\begin{array}{c}\text { Material de baixo cimento a base de alumina } \\
\text { e carbeto de silício }\end{array}$ \\
\hline
\end{tabular}

A atividade de aplicação do refratário foi definida em duas etapas: a cúpula do topo com projeção manual e a coroa de choque e cubas por meio robotizado.

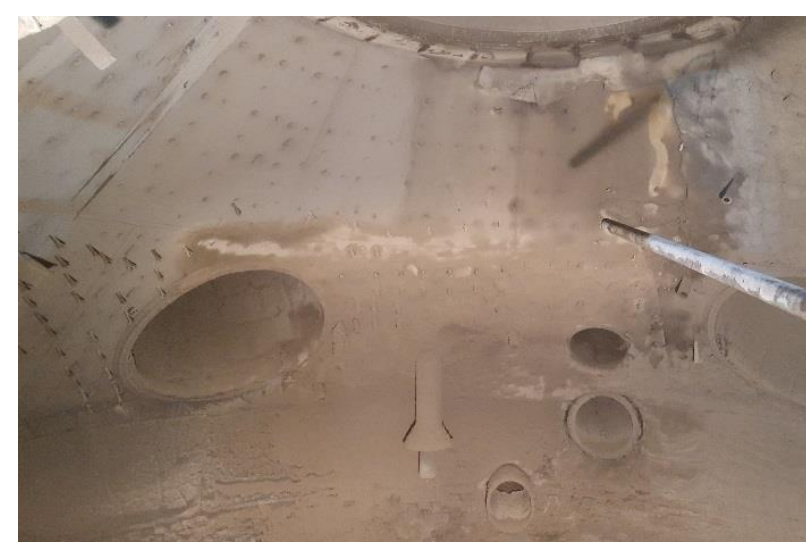

a) projeção manual na cúpula de topo

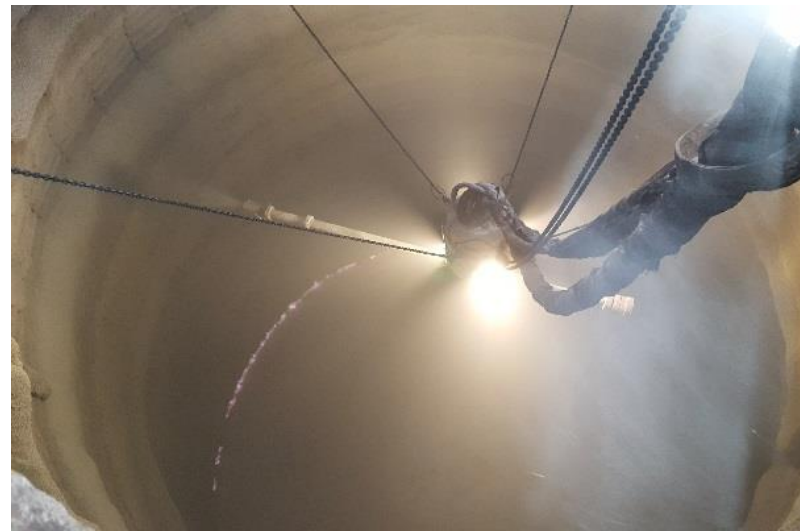

b) projeção robotizada na coroa de choque e cuba

Figura 2. Atividades de reperfilamento do refratário do Alto-Forno 2

\subsection{Planejamento de Reparo e Execução}

O planejamento das atividades referentes ao shotcrete do Alto-Forno 2 consistiu nas atividades que deveriam ser executadas com o forno em operação, nas atividades que necessitavam do forno parado, abaixamento de carga e projeção de refratário. 
As atividades que foram realizadas com o forno em operação consistiam basicamente de preparativos operacionais, treinamentos, aspectos de segurança operacional, adaptação e adequação de componentes e ajustes de composição química dos materiais pertencentes a carga metálica.

Atividades que foram executadas na parada preparatória para o shotcrete ocorrida no dia 26/03/2017:

- Inspeção da região de reparo na cúpula do topo;

- Instalação de tomadas de $\mathrm{N}_{2}$ no corpo do Alto-Forno 2;

- Instalar sistema de umectação do coque a ser carregado;

- Instalação de sistema de medição de gases $\left(\mathrm{CO}, \mathrm{CO}_{2}\right.$ e $\left.\mathrm{H}_{2}\right)$ em faixas especiais e sistema de análise de $\mathrm{O}_{2}$;

- Substituição dos bicos de spray da carga;

- Substituição das ventaneiras com bico danificado;

- Instalação de cigar coolers;

- Retirada de sonda fixa danificada.

No abaixamento de carga, o forno foi operado com leito allcoke (1000kg/t) e sem coque médio. O nível de carga planejado era de $18 \mathrm{~m}$ que correspondia a parte inferior da terceira fileira de staves de cobre, permitindo o acesso do robô ao interior do forno para a projeção na face quente dos staves. No final do procedimento, a carga se encontrava a $18,15 \mathrm{~m}$ do stock line com o tempo total da descida de carga de 10h20min.

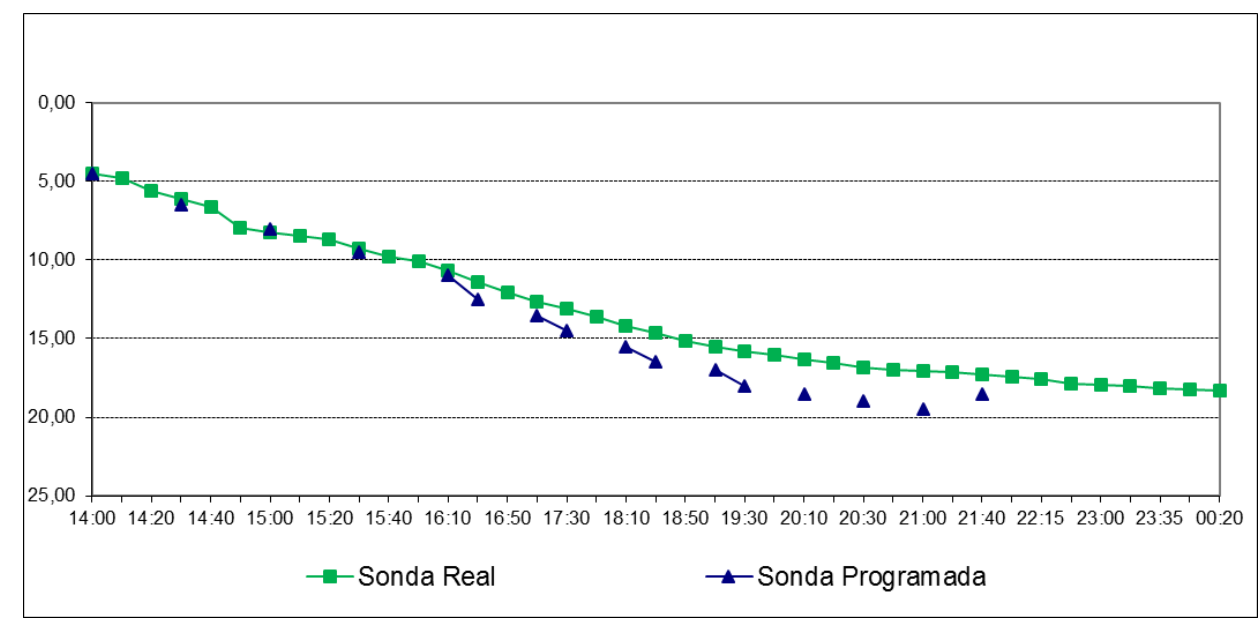

Figura 3. Acompanhamento sonda real x sonda programada

Ao longo de todo processo de abaixamento foram controladas criteriosamente as seguintes variáveis operacionais: vazão de ar soprado, nível de sonda mecânica, nível de sonda radar, temperatura de topo, pressão de topo, pressão de sopro, delta $\mathrm{P}$, índice de permeabilidade, temperatura de sopro e análise de gases.

Após o abaixamento de carga foi aplicado o material isolante com a finalidade de proteger o robô da radiação térmica da carga, e posteriormente, material para favorecer a descompactação do rebote foi lançado para o interior do Alto-Forno. 


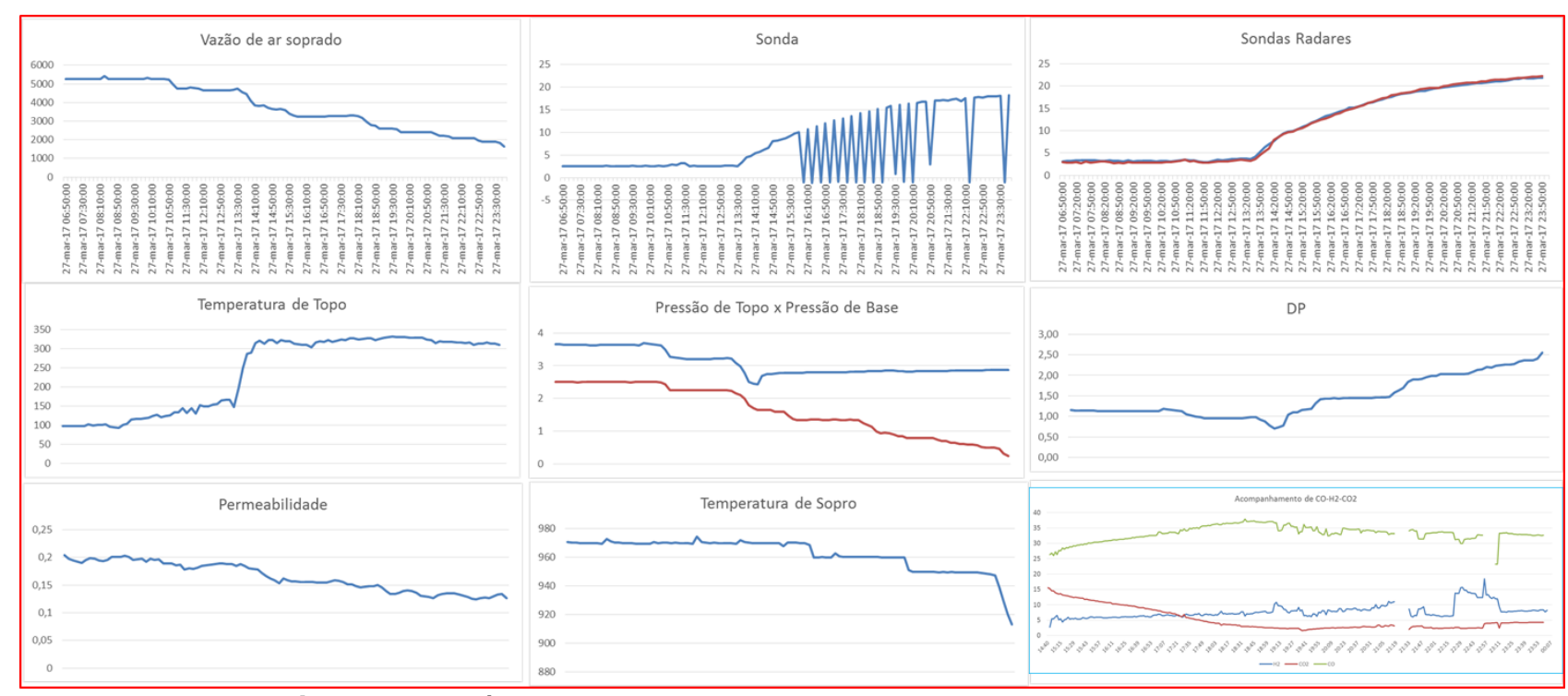

Figura 4. Variáveis operacionais durante o abaixamento de carga

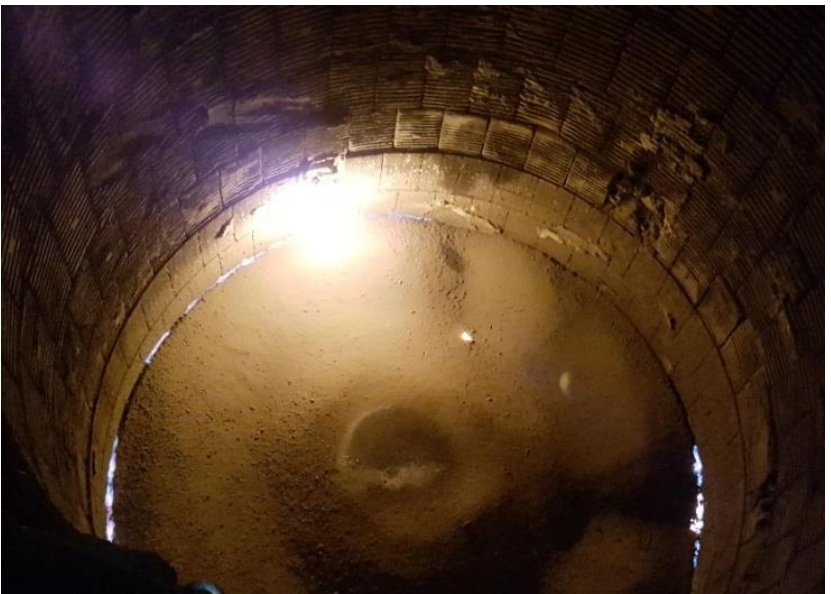

a) Burden capping

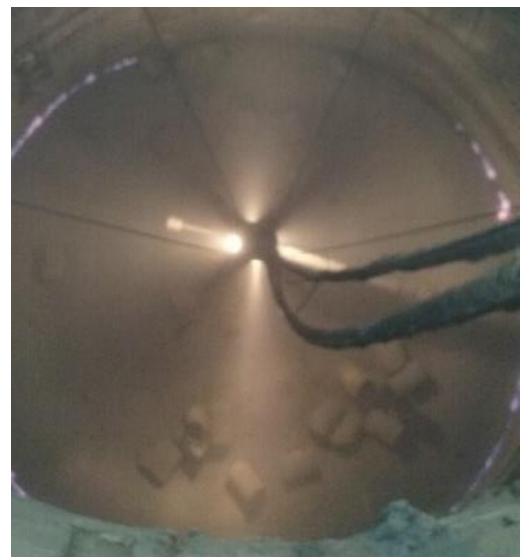

b) Tambores para descompactação do rebote

Figura 5. Atividades realizadas antes da aplicação do refratário

O total de material refratário utilizado no processo de projeção de refratário foi de 207 toneladas. E o rebote gerado durante o processo foi de aproximadamente $17,5 \%$, calculado pelo balanço de alumina na escória, correspondente a $36 t$ de massa refratária.

O retorno de operação do Alto-Forno 2, no dia 01 de abril, foi realizado sem dificuldades. A primeira corrida não teve problemas na perfuração do furo de gusa e as condições operacionais padrão foram estabelecidas com facilidade. 


\section{RESULTADOS E DISCUSSÃO}

Após a execução do shotcrete, o Alto-Forno 2 apresentou elevação considerável na produção diária, em torno de $470 \mathrm{t} / \mathrm{d}$, principalmente devido a melhora na estabilidade operacional do equipamento que favoreceu aos ganhos observados.

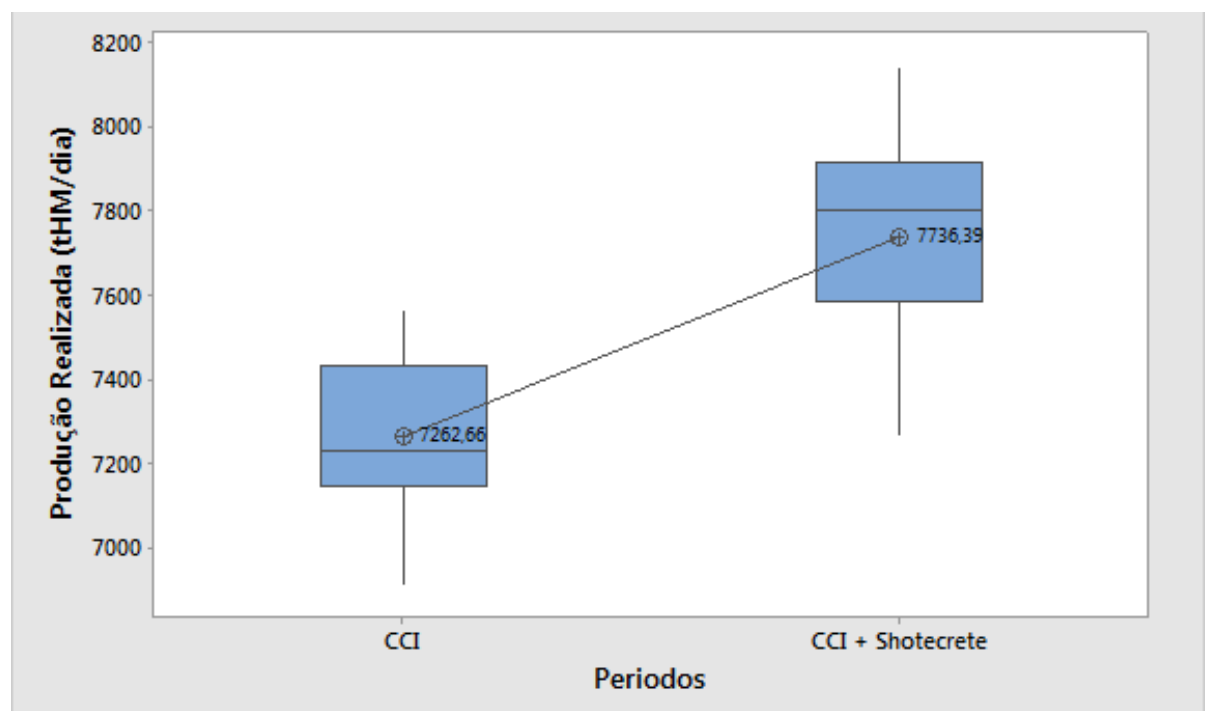

Figura 6. Verificação da diferença de produção por alteração na distribuição de carga $x$ shotcrete

Em paralelo a esta melhora de produção também foi observado um ganho no fuel rate, em torno de $18 \mathrm{~kg} / \mathrm{t}$ de redução. Esta redução foi influenciada também pela estabilidade alcançada pelo conjunto de ações de distribuição de carga e shotcrete, sendo que o valor atribuído para este último é $10 \mathrm{~kg} / \mathrm{t}$.

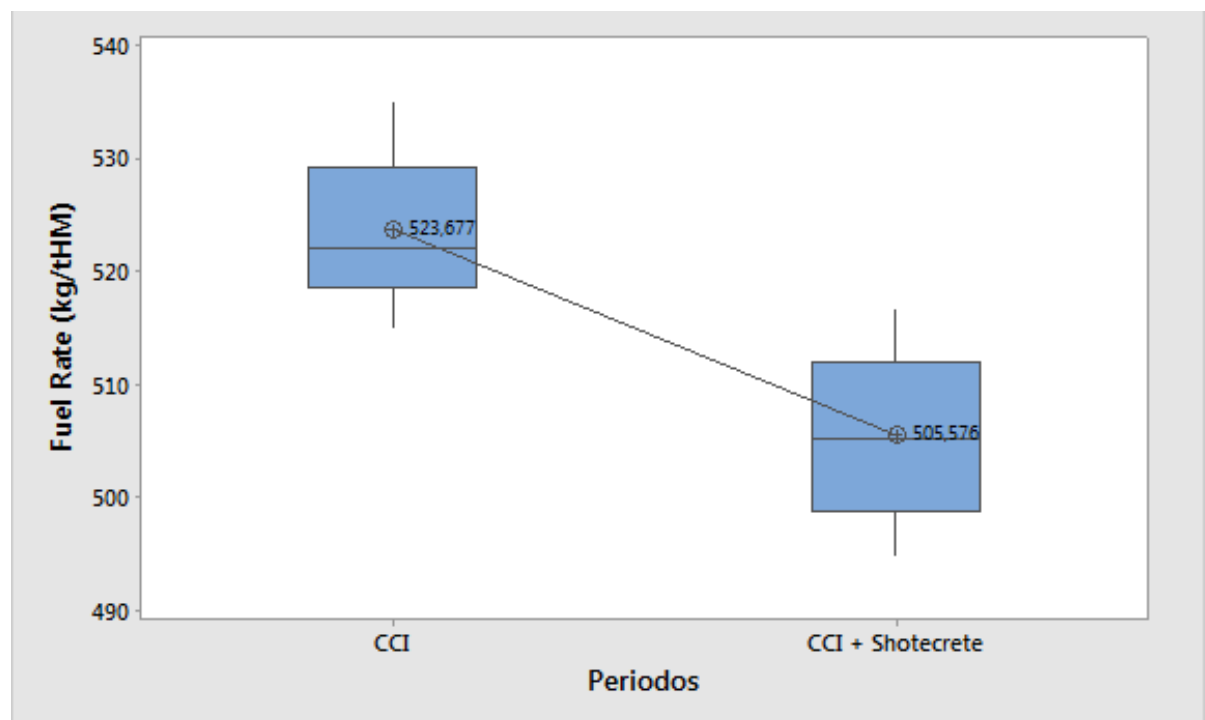

Figura 7. Verificação da diferença de fuel rate por alteração na distribuição de carga x shotcrete 


\section{CONCLUSÃO}

A realização do shotcrete foi importante para a evolução do Alto-Forno 2, onde foi atribuído um aumento de eficiência com redução de $10 \mathrm{~kg} / \mathrm{t}$ no fuel rate, sendo metade na redução da carga térmica e a outra metade por efeitos indiretos como a melhor distribuição na coroa de choque.

Devido a estabilização do forno pelos efeitos indiretos, observamos um aumento significativo na produção de gusa, onde $50 \%$ desse aumento pode ser atribuído ao shotcrete

Considerando os ganhos diretos e indiretos, de forma conservadora, o retorno de investimento foi estimado em 0,14 anos. Os ganhos na vida útil do forno não foram considerados nesta estimativa.

A verificação da necessidade de nova realização do shotcrete será realizada observando o comportamento após a primeira aplicação, a estimativa é de aplicação anual de massa refratária. O comportamento do shotcrete que será realizado no Alto-Forno 1, provavelmente, não terá os mesmos resultados deste realizado, pois os fornos não possuem as mesmas condições operacionais e nem apresentam as mesmas condições de preservação dos staves.

\section{REFERÊNCIAS}

1 BABICH, A.; SENK, D.; GUDENAU,H.W.;MAVROMMATIS.K.TH. - Ironmaking Textbook - Aachen: RWTH; 2008. 